\title{
On disjoint arithmetic progressions
}

\author{
by \\ Yong-Gao Chen (Nanjing)
}

1. Introduction. Let $\left\{a_{i}\left(\bmod m_{i}\right)\right\}_{i=1}^{l}$ be a collection of arithmetic progressions, where $2 \leq m_{1}<\cdots<m_{l} \leq x$ and

$$
a_{i}\left(\bmod m_{i}\right) \cap a_{j}\left(\bmod m_{j}\right)=\emptyset \quad \text { if } i \neq j .
$$

Let $f(x)$ be the maximum value for such $l$. In [4], Erdős and Szemerédi proved that

$$
\frac{x}{\exp \left((\log x)^{1 / 2+\varepsilon}\right)}<f(x)<\frac{x}{(\log x)^{c}}
$$

for some constant $c>0$ (see also [3]). Let

$$
L(c, x)=\exp (c \sqrt{\log x \log \log x}) .
$$

Recently, E. S. Croot III [2] has proved that

$$
\frac{x}{L(\sqrt{2}+o(1), x)}<f(x)<\frac{x}{L(1 / 6-o(1), x)}
$$

and with the assumption that all $m_{i}$ are squarefree, the maximum value for such $l$ is less than $x / L(1 / 2-o(1), x)$.

In this paper, we improve Croot's methods and prove the following result:

THEOREM.

$$
f(x)<\frac{x}{L(1 / 2-o(1), x)} .
$$

2. Proof of the Theorem. As a direct consequence of [1, Lemma 3.1], we have (see also [2])

2000 Mathematics Subject Classification: Primary 11B25.

Supported by the National Natural Science Foundation of China, Grant No.10471064 and the Teaching and Research Award Program for Outstanding Young Teachers at Nanjing Normal University. 
LEMma 1. Let

$$
\psi(x, y)=\#\{n \leq x: p \text { prime }, p \mid n \Rightarrow p \leq y\} .
$$

Then, for any constant $c>0$,

$$
\psi(x, L(c, x))=\frac{x}{L(1 /(2 c)+o(1), x)} .
$$

LEMMA 2 (Croot [2]). There are at most $x / L(c / 2-o(1), x)$ positive integers $n \leq x$ such that

$$
\omega(n)>c \sqrt{\log x / \log \log x}
$$

where $c$ is some positive constant and $\omega(n)=\sum_{p \mid n, p \text { prime }} 1$.

Definition. Let $r$ and $n$ be positive integers. Write

$$
n=a b, \quad(a, b)=1, \quad a>0, b>0,
$$

such that if $p$ is a prime, $p^{\alpha} \mid a$ and $p^{\alpha+1} \nmid a$, then either $\alpha=0$ or $\alpha>r$, and if $p$ is a prime, $p^{\beta} \mid b$ and $p^{\beta+1} \nmid b$, then $\beta \leq r$. Define

$$
h_{r}(n)=a, \quad l_{r}(n)=b .
$$

Here we may take $a=1$ or $b=1$.

LEMma 3. Let $r$ be a given positive integer. Then there are at most $x / L(c / 2-o(1), x)$ positive integers $n \leq x$ with $n=l_{r}(n)$ such that

$$
\Omega(n)>c \sqrt{\log x / \log \log x}
$$

where $c$ is some positive constant and $\Omega(n)=\sum_{p^{\alpha} \mid n, p \text { prime }} 1$.

Proof. Let

$$
\begin{aligned}
& T_{1}=\{n: n \leq x, \omega(n)>c \sqrt{\log x / \log \log x}\} \\
& T_{2}=\left\{n: n \leq x, n=l_{r}(n), \omega(n) \leq c \sqrt{\log x / \log \log x},\right. \\
& \\
&\quad \Omega(n)>c \sqrt{\log x / \log \log x}\} .
\end{aligned}
$$

By Lemma 2 we have

$$
\left|T_{1}\right| \leq \frac{x}{L(c / 2-o(1), x)}
$$

Now we estimate $\left|T_{2}\right|$. For a positive integer $n$, let $n=p_{1}^{\alpha_{1}} \cdots p_{t}^{\alpha_{t}}$ be the standard factorization of $n$. Define

$$
g(n)=\alpha_{1} ! \cdots \alpha_{t} !
$$

By a multipolynomial expansion, we have

$$
\left(\sum_{p \leq x, p \text { prime }} \frac{1}{p}\right)^{j} \geq \sum_{n \leq x, \Omega(n)=j} \frac{j !}{g(n) n} .
$$


Thus

$$
\begin{aligned}
\sum_{n \leq x, \Omega(n) \geq c \sqrt{\log x / \log \log x}} \frac{1}{g(n) n} & \leq \sum_{j \geq c \sqrt{\log x / \log \log x}} \frac{1}{j !}\left(\sum_{p \leq x, p \text { prime }} \frac{1}{p}\right)^{j} \\
& \leq \frac{1}{L(c / 2-o(1), x)} .
\end{aligned}
$$

For any integer $n$ with $n \in T_{2}$, we have

$$
g(n) \leq(r !)^{\omega(n)} \leq(r !)^{c \sqrt{\log x / \log \log x}} .
$$

Hence

$$
\left|T_{2}\right| \leq \sum_{n \in T_{2}} \frac{x}{n} \leq x(r !)^{c \sqrt{\log x / \log \log x}} \sum_{n \in T_{2}} \frac{1}{g(n) n} \leq \frac{x}{L(c / 2-o(1), x)} .
$$

Lemma 4. For any integer $k \geq 3$, we have

$$
\#\left\{n: 1 \leq n \leq x, h_{k(k+1)}(n) \geq L(c, x)\right\} \leq \frac{3 x}{L(c(1-2 / k), x)} .
$$

Proof. It is well known that if an integer $\alpha>k(k+1)$, then there exist two positive integers $u$ and $v$ with $\alpha=u k+v(k+1)$. Thus, if $m=h_{k(k+1)}(m)$, then there exist two positive integers $a$ and $b$ such that $m=a^{k} b^{k+1}$. So

$$
S_{k(k+1)}(t):=\#\left\{m: 1 \leq m \leq t, m=h_{k(k+1)}(m)\right\} \leq t^{\frac{1}{k}+\frac{1}{k+1}}<t^{2 / k} .
$$

Hence

$\#\left\{n: 1 \leq n \leq x, h_{k(k+1)}(n) \geq L(c, x)\right\}$

$$
\leq \sum_{m \geq L(c, x), m=h_{k(k+1)}(m)} \frac{x}{m}=x \int_{L(c, x)}^{\infty} \frac{1}{t} d S_{k(k+1)}(t) \leq \frac{3 x}{L(c(1-2 / k), x)} \text {. }
$$

Lemma 5. Let $r^{\prime}$ and $m$ be two integers with $m>0$. Suppose that $\left\{b_{i}\left(\bmod m_{i}\right)\right\}_{i=1}^{l}$ is a collection of disjoint arithmetic progressions, where

$$
\begin{gathered}
\omega\left(m_{i}\right) \leq M, \quad m_{i}>m, \\
m \mid m_{i}, \quad b_{i} \equiv r^{\prime}(\bmod m), \quad i=1, \ldots, l .
\end{gathered}
$$

Then there exists a prime $p$ and an integer $r^{\prime \prime}$ such that $p m \mid m_{i}$ for at least $l / M$ values of $i$, and

$$
p m \mid m_{i}, \quad b_{i} \equiv r^{\prime \prime}(\bmod p m)
$$

for at least $l /(p M)$ values of $i$.

Proof. If $l=1$, then we may take $r^{\prime \prime}=b_{1}$ and $p$ to be any prime divisor of $m_{1} / m$. Now we assume that $l \geq 2$. Let $p_{1}, \ldots, p_{t}$ be all distinct prime factors of $m_{1} / m$. For any $i \geq 2$, since the arithmetic progressions are disjoint, we have $\left(m_{1}, m_{i}\right) \nmid b_{1}-b_{i}$. By $b_{1} \equiv r^{\prime} \equiv b_{i}(\bmod m)$, we have $\left(m_{1}, m_{i}\right) \neq m$. That is, $\left(m_{1} / m, m_{i} / m\right) \neq 1(i=2, \ldots, l)$. Hence, each $m_{i} / m$ must be divisible by 
at least one of $p_{1}, \ldots, p_{t}$. Thus, there exists an index $j$ such that $p_{j}$ divides $m_{i} / m$ for at least $l / t$ values of $i$. Let $b_{i}=r^{\prime}+m s_{i}(i=1, \ldots, l)$. Then there exists an integer $s$ such that

$$
s_{i} \equiv s\left(\bmod p_{j}\right), \quad p_{j} \mid \frac{m_{i}}{m}
$$

for at least $l /\left(p_{j} t\right)$ values of $i$. Thus, by $t \leq M, p_{j}$ divides $m_{i} / m$ for at least $l / M$ values of $i$, and

$$
b_{i} \equiv r^{\prime}+m s\left(\bmod p_{j} m\right), \quad p_{j} m \mid m_{i}
$$

for at least $l /\left(p_{j} M\right)$ values of $i$.

Lemma 6. Let $r$ be a given positive integer. If $\left\{b_{i}\left(\bmod m_{i}\right)\right\}_{i=1}^{l}$ is a collection of disjoint arithmetic progressions with $m_{i}=l_{r}\left(m_{i}\right)(i=1, \ldots, l)$ and $2 \leq m_{1}<\cdots<m_{l} \leq x$, then

$$
l<\frac{x}{L(1 / 2-o(1), x)} .
$$

Proof. Let $S$ be the collection of all $m_{i}$ 's such that

(A) $\Omega\left(m_{i}\right) \leq \sqrt{\log x / \log \log x}$;

(B) There exists a prime $p>L(1, x)$ such that $p \mid m_{i}$.

Beginning with $m=1$ and $r^{\prime}=1$, by using Lemma 5 step by step, we obtain a sequence $p_{1}, \ldots, p_{v}$ of primes (and a sequence $r_{1}, \ldots, r_{v}$ of integers) such that for each $j, p_{1} \cdots p_{j} \mid m_{i}$ for at least

$$
\frac{|S|}{p_{1} \cdots p_{j-1}(\sqrt{\log x / \log \log x})^{j}}
$$

values of $i$ with $m_{i} \in S$ and $p_{1} \cdots p_{v}$ is some $m_{i_{0}}$. Hence, there exists a $p_{u}$ with $p_{u}>L(1, x)$, and

$$
\begin{aligned}
\frac{x}{p_{1} \cdots p_{u-1} L(1, x)} & \geq \#\left\{n \leq x: p_{1} \cdots p_{u} \mid n\right\} \\
& \geq \#\left\{m_{i}: m_{i} \in S, p_{1} \cdots p_{u} \mid m_{i}\right\} \\
& \geq \frac{|S|}{p_{1} \cdots p_{u-1}(\sqrt{\log x / \log \log x})^{u}} \\
& \geq \frac{|S|}{p_{1} \cdots p_{u-1} L(1 / 2, x)} .
\end{aligned}
$$

Thus

$$
|S| \leq \frac{x}{L(1 / 2, x)}
$$


By Lemmas 1 and 3, we have

$$
\begin{aligned}
|S| & \geq l-\#\left\{m \leq x: m=l_{r}(m), \Omega(m)>\sqrt{\log x / \log \log x}\right\}-\psi(x, L(1, x)) \\
& \geq l-\frac{2 x}{L(1 / 2-o(1), x)} .
\end{aligned}
$$

Therefore

$$
l \leq \frac{x}{L(1 / 2-o(1), x)}
$$

Proof of the Theorem. Suppose that $\left\{a_{i}\left(\bmod m_{i}\right)\right\}_{i=1}^{f(x)}$ is a collection of disjoint arithmetic progressions, where $2 \leq m_{1}<\cdots<m_{f(x)} \leq x$. Let $k$ be any given integer with $k \geq 4$, and let $U$ be the collection of all $m_{i}$ with $h_{k(k+1)}\left(m_{i}\right)<L(2, x)$. By the proof of Lemma 4, we have

$$
\#\left\{m: 1 \leq m \leq L(2, x), m=h_{k(k+1)}(m)\right\} \leq L(2, x)^{2 / k}=L(4 / k, x) .
$$

So there must exist a positive integer $m<L(2, x)$ for which there are at least $|U| / L(4 / k, x)$ of $m_{i}$ 's with $h_{k(k+1)}\left(m_{i}\right)=m$. Thus, there must exist an integer $a$ such that

$$
a_{i} \equiv a(\bmod m), \quad h_{k(k+1)}\left(m_{i}\right)=m
$$

for at least $|U| /(m L(4 / k, x))$ of $m_{i}$. In this way, we obtain a collection $\left\{a_{i}\left(\bmod m_{i} / m\right)\right\}$ which has at least $|U| /(m L(4 / k, x))$ disjoint arithmetic progressions. Noting that $l_{k(k+1)}\left(m_{i} / m\right)=m_{i} / m$ for those $m_{i}$ with $h_{k(k+1)}\left(m_{i}\right)=m$, by Lemma 6 , we have

$$
\frac{|U|}{m L(4 / k, x)} \leq \frac{x}{m L(1 / 2-o(1), x / m)} \leq \frac{x}{m L(1 / 2-o(1), x)} .
$$

So

$$
|U| \leq \frac{x}{L(1 / 2-4 / k-o(1), x)} .
$$

By Lemma 4 we have

$$
\#\left\{n: 1 \leq n \leq x, h_{k(k+1)}(n) \geq L(2, x)\right\} \leq \frac{3 x}{L(2(1-2 / k), x)} \leq \frac{3 x}{L(1, x)} .
$$

Therefore

$$
f(x) \leq \frac{x}{L(1 / 2-4 / k-o(1), x)}+\frac{3 x}{L(1, x)} .
$$

Since $k$ is arbitrary, the Theorem follows.

Acknowledgements. I am grateful to Professor E. S. Croot III for his help to make me understand his paper and the referee for his/her suggestions. 


\section{References}

[1] E. R. Canfield, P. Erdős and C. Pomerance, On a problem of Oppenheim concerning "factorisatio numerorum", J. Number Theory 17 (1983), 1-28.

[2] E. S. Croot III, On non-intersecting arithmetic progressions, Acta Arith. 110 (2003), 233-238.

[3] P. Erdős and R. L. Graham, Old and New Problems and Results in Combinatorial Number Theory, Monograph. Enseign. Math. 28, Univ. Genève, 1980.

[4] P. Erdős and E. Szemerédi, On a problem of P. Erdős and S. Stein, Acta Arith. 15 (1968), 85-90.

Department of Mathematics

Nanjing Normal University

Nanjing 210097, P.R. China

E-mail: ygchen@njnu.edu.cn

Received on 23.2.2004

and in revised form on 13.2.2005 